\title{
Effet d'un déficit hydrique sur le trèfle blanc (Trifolium repens L.). I. Importance du cultivar
}

\author{
L. Shamsun Noor ${ }^{-1}$, C. Robin ${ }^{1}$, D. Schontz ${ }^{1}$, T. Heulin ${ }^{2}$ et A. Guckert ${ }^{1}$ \\ 1 Laboratoire de Phytotechnie, INRA, ENSAIA, 2, avenue de la Forêt-de-Haye, 54500 Vandcuuvre; \\ 2 LEMIR, CPB, CNRS, rue Notre-Dame-des-Pauvres, 54500 Vandœuvre, France
}

(reçu le 12-1-1988, accepté le 12-12-1988)

\begin{abstract}
Résumé - Quatre cultivars de trèfle blanc(Trifolium repens L., Ladino ou Hollandicum) ont été soumis à un déficit hydrique croissant provoqué par l'arrêt total de l'alimentation en eau. L'expérience a été effectuée, en chambre phytotronique, 9 semaines après la germination des plantes. Le déficit en eau entraîne une diminution rapide du potentiel hydrique de base et de l'assimilation photosynthétique nette pour tous les cultivars.

Cette étude confirme aussi le lien entre le facteur hydrique et la fixation d'azote, dont l'optimum n'est pas atteint à la capacité au champ, sans doute en raison d'une aération insuffisante du sol à cette humidité, mais correspond à une teneur en eau du sol comprise entre 60 et $70 \%$ de la capacité au champ pour le type Ladino et entre 80 et $85 \%$ pour le type Hollandicum. Des différences intervariétales ont été mises en évidence : Crau (type Ladino) maintient le taux de fixation le plus élevé et produit plus de biomasse aérienne que les autres variétés. Le cultivar Huia (type Hollandicum) présentant la fixation symbiotique la plus basse se montre le moins tolérant au déficit hydrique.

L'intérêt de cette étude est discuté dans une optique de criblage de cultivars, sur la base de leur tolérance au manque d'eau.
\end{abstract}

\section{Ladino - Hollandicum - potentiel hydrique - photosynthèse - fixation de l'azote}

Summary - Effect of water deficit on white clover. I. Importance of the cultivar. Four white clover cultivars (Trifolium repens $L$. Ladino and Hollandicum) were grown in pots placed in a growth chamber. Nine weeks after germination the water supply was suppressed. Dawn water potential, net photosynthetic assimilation and nitrogen fixation were measured. The water stress resulted in a rapid decrease in the dawn water potential. Photosynthesis and nitrogen fixation were also affected.

The relationship between nitrogen fixation and soil water content was confirmed. The maximum rate of nitrogen fixation was not reached at the soil water capacity, probably due to a lack of soil aeration. Nitrogen fixation was maximum at $60-70 \%$ and $80-85 \%$ of the soil water capacity with Ladino and Hollandicum respectively. A lack of aeration at the water holding capacity probably caused an inhibition of the nitrogen fixation. Differences in resistance to water deficit were observed between varieties : Crau (Ladino) presented the highest nitrogen fixation, and produced more biomass than other varieties. Huia (Hollandicum) showed the lowest nitrogen fixation and appeared to be the most sensitive variety.

Methods for selecting cultivars as regard to their resistance or tolerance to water deficit are discussed.

Ladino - Hollandicum - water potential - photosynthesis - nitrogen fixation

\section{Introduction}

Le trèfle blanc, légumineuse fourragère pérenne, représente une espèce importante de nos prairies permanentes. II constitue également, du fait de son aptitude à fixer par voie symbiotique l'azote atmosphérique, un partenaire intéressant dans les associations graminées - légumineuses. Le rendement fourrager de telles associations est grandement conditionné par l'alimentation en eau. Or, c'est pendant la phase estivale, souvent marquée par des déficits hydriques, que le trèfle blanc est à même d'exprimer son potentiel de production, alors que la compétition interspécifique est moindre. (Laissus, 1981). Peu d'études ont été conduites chez les légumineuses pérennes, et notamment chez le trèfle blanc, quant à leur comportement face à un déficit hydrique (Engin et Sprent, 1973; Foulds, 1978). Guckert et Laperrière (1987) ont montré l'inciden- 
ce du déficit hydrique associé à la fertilisation potassique au niveau de la fixation symbiotique de l'azote. Le présent travail vise à mettre en évidence le rôle du facteur variétal chez le trèfle blanc en situation de stress hydrique; 4 variétés : 2 de type Hollandicum et 2 de type Ladino ont ainsi été comparées.

Trois paramètres essentiels ont été suivis afin de rendre compte de l'effet d'un déficit hydrique: - le statut hydrique de la plante (par la détermination de son potentiel hydrique);

- la photosynthèse (assimilation nette);

- la fixation d'azote, appréhendée par la mesure de l'activité réductrice d'acétylène de la nitrogénase (ARA).

\section{Matériel et Méthodes}

\section{Matériel végétal et conditions de culture}

L'ensemble de l'expérimentation a concerné 4 variétés de trèfle blanc, correspondant à 2 types différents :

- type Hollandicum: Huia et Luclair;

- type Ladino: Crau et Major.

Chaque variété a été semée en vase de végétation (hauteur $=25 \mathrm{~cm}$ ), avec 15 plantes par pot correspondant à une densité de 450 plantes $\mathrm{m}^{-2}$ sur un sol argileux (sol brun calcaire marmorisé à $40 \%$ d'argiles) provenant du domaine expérimental de la Bouzule et ayant reçu une fertilisation phospho-potassique équivalente à $P, K(200,200)$ U.ha-1.

Pendant les 7 semaines suivant le semis, les plantes séjournent en serre thermorégulée $\left(28^{\circ} \mathrm{C}\right.$ le jour et 20 à $22^{\circ} \mathrm{C}$ la nuit); elles subissent un arrosage quotidien maintenant la teneur en eau du sol à la capacité au champ, qui correspond à $26 \%$ d'humidité pondérale. Pour la réalisation du déficit hydrique, les cultivars sont transtérés en cellule phytotronique (thermopériode $21^{\circ} \mathrm{C}$ le jour, $18^{\circ} \mathrm{C}$ la nuit, photopériode de $14 \mathrm{~h}$, intensité lumineuse de l'ordre de $300 \mu \mathrm{mol} \cdot \mathrm{m}^{-2} \cdot \mathrm{s}^{-}$ 1 , humidité relative $76 \%$.

Une semaine après le transfert, les plantes correspondant à chaque cultivar sont divisées en 2 lots (avec 4 répétitions par lot) :

- un lot témoin maintenu à la capacité au champ;

- un lot soumis au déficit hydrique : suppression de l'alimentation en eau durant l'expérience.

\section{Méthodes}

\section{Mesure du potentiel hydrique des feuilles}

Selon Aussenac et Granier (1978), l'état de liaison de l'eau dans la plante, ou potentiel hydrique, est sous la dépendance de 3 composantes:

-2 facteurs externes, la contrainte climatique et la disponibilité en eau du sol;

- 1 facteur biologique interne, la réponse de la plante qui réalise la jonction entre la demande en eau et l'offre du sol.

Ritchie et Hinckley (1975) ont défini le potentiel de base comme le potentiel d'équilibre entre la plante et le sol lorsque la transpiration est nulle; il est atteint en fin de nuit. II reflète donc le potentiel de l'eau dans le sol.

Le potentiel hydrique est mesuré à la chambre à pression de Schölander (Schölander et al., 1965) sur des feuilles de même rang initiées par les pieds mères.

\section{Mesure de la fixation symbiotique de l'azote}

La fixation symbiotique de l'azote est estimée par la méthode indirecte de mesure de l'activité réductrice d'acétylène ou méthode ARA (Koch et Evans, 1966). Le dispositif utilisé est une adaptation au trèfle blanc (Guckert et al., 1983) de la méthode proposée par Balandreau et Dommergues (1973). Le dosage de l'acétylène produit après l'incubation se fait à l'aide d'un chromatographe en phase gazeuse muni d'un détecteur à ionisation de flamme et d'une colonne Sphérosyl XOB $075,10 \% \mathrm{Na}_{3} \mathrm{PO}_{4}$, avec de l'azote comme gaz vecteur (pression de 2 bars).

L'ARA est exprimée en moles d'éthylène produites par heure d'incubation et par gramme de matière sèche de parties aériennes. Chez le trèfle blanc, il a été clairement démontré l'existence d'une corrélation étroite entre I'ARA exprimée par g MS de parties aériennes et l'ARA exprimé par g MF de nodosités (Damay, 1981; Laperrière, 1984).

\section{Mesure de la photosynthèse}

Le dispositif expérimental de mesure de I'ARA (cloches en polyéthylène) permet de suivre l'évolution des activités photosynthétiques et respiratoires de plantes dans une enceinte close. Les plants de trèfle sont placés dans un premier temps à l'obscurité (accroissement de la teneur en $\mathrm{CO}_{2}$ de l'enceinte par respiration), puis exposés rapidement à la lumière. II en résulte une absorption hyperbolique du $\mathrm{CO}_{2}$ au cours du temps. On réalise à des intervalles de temps réguliers des prélèvements de gaz de l'atmosphère interne, dont on détermine la teneur en $\mathrm{CO}_{2}$ à l'aide d'un analyseur infrarouge (Cosma Rubis 3000). Pour une teneur donnée en $\mathrm{CO}_{2}$ (en général celle au niveau du couvert), l'assimilation nette (AN) est exprimée par la valeur en ce point de la tangente à la courbe de décroissance du $\mathrm{CO}_{2}$ (Damay, 1981). Les résultats d'assimilation sont donnés en $\mathrm{mg}$ de $\mathrm{CO}_{2}$ assimilé par heure d'incubation et par pot.

\section{Traitement des données}

L'étude statistique des données s'appuie sur des analyses de variance et des comparaisons des moyennes selon le test de Newman-Keuls. Les mesures de potentiel hydrique de base sont les moyennes de 8 répétitions, les mesures d'ARA sont les moyennes de 4 répétitions, celles d'assimilation nette sont les moyennes de 3 répétitions.

\section{Résultats}

\section{Evolution du potentiel hydrique de base}

Dans le cas des traitements "témoins", normalement pourvus en eau, on n'observe pas de différences significatives $(P<0,05)$ pour le potentiel de base entre les 4 cultivars (Fig. 1); les valeurs évoluent entre -1 et -2 bars. 


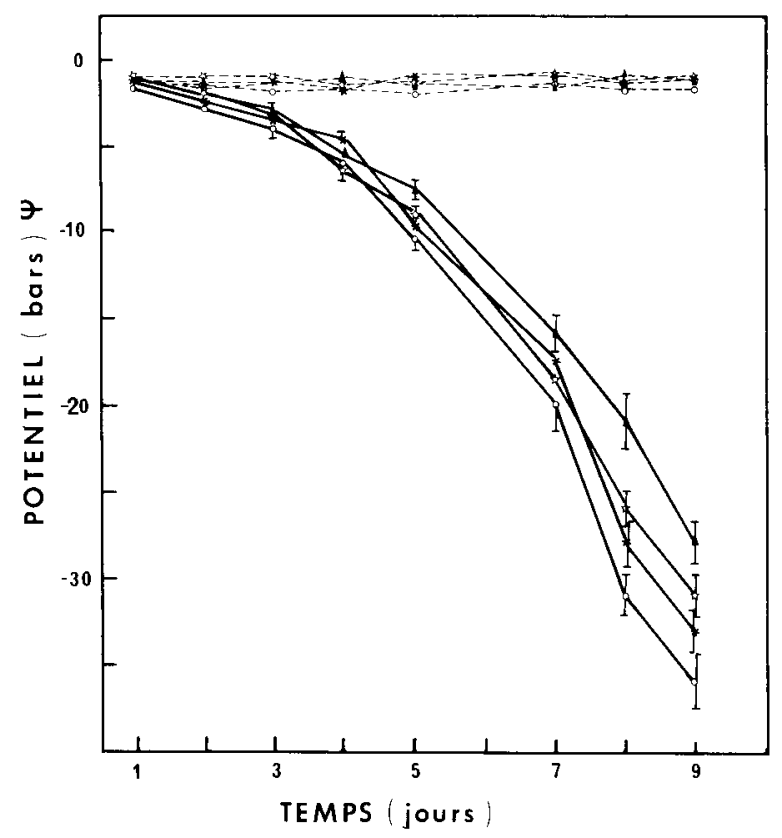

Fig. 1. Evolution du potentiel de base (bars) en fonction du temps pour 4 cultivars de trèfle blanc : Crau $(\boldsymbol{A})$, Major ( Luclair (洸) et Huia (O), subissant ( - ) ou non (- - - ) un déficit hydrique. Les traits verticaux correspondent à l'écart type des mesures.

Pour les plantes soumises à un stress hydrique sévère, caractérisé par la diminution de l'humidité du sol (Fig. 2), le potentiel de base décroît régulièrement jusqu'au $9 \mathrm{e}$ jour, stade où le point de flétrissement est atteint. Les valeurs mesurées atteignent alors en moyenne -32 bars pour l'ensemble des cultivars en situation de déficit. A cette date, une différence significative de 8 bars sépare Crau de Huia.

L'analyse de variance (données non produites) indique un effet significatif $(P<0,05)$ du facteur «déficit» dès le $2^{e}$ jour suivant l'arrêt de l'arrosage. La courbe de potentiel hydrique de base du cultivar Huia chute plus rapidement que celle des 3 autres. En fin d'expérience, le classement des variétés suivant leur potentiel hydrique $\psi$ est le suivant : $\psi$ Crau $>\psi$ Major $>\psi$ Luclair $>$ $\psi$ Huia.

\section{Activité photosynthétique}

L'activité photosynthétique des lots "témoins" est sujette à des variations inhérentes à la méthode de mesure, elle-même sensible aux conditions phytotroniques (gradient lumineux) et vraisemblablement à la variation de teneur en $\mathrm{CO}_{2}$ de la chambre de végétation par apport de $\mathrm{CO}_{2}$ respiratoire (manipulateur).

Lorsque les plantes sont soumises au déficit hydrique, l'activité photosynthétique diminue brutalement dès le $2^{\mathrm{e}}$ jour pour atteindre des valeurs proches de 0 lors du 7 e jour. Lorsque la variabilité

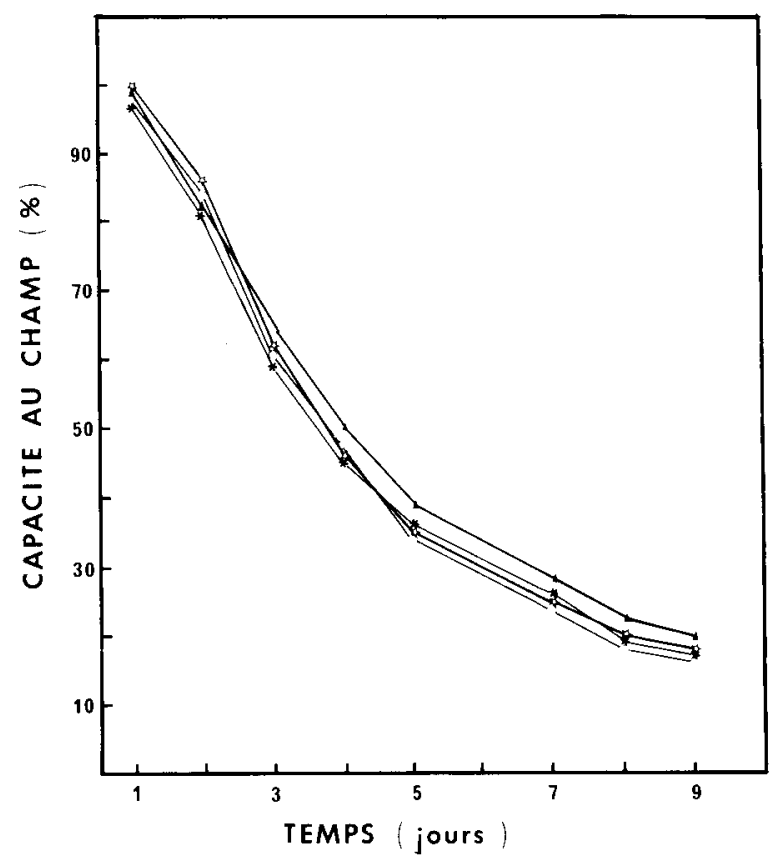

Fig. 2. Evolution de l'humidité du sol (\% de la capacité au champ) en fonction du temps (jours) pour les 4 cultivars de

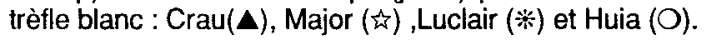

des résultats permet de mettre en évidence des différences significatives $(P<0,01,4 \mathrm{e}$ jour $)-$ (Tableau I), le classement des cultivars est le même que précédemment : Crau > Major > Luclair > Huia.

Au $4^{e}$ jour d'arrêt de l'irrigation, l'humidité du sol atteint $50 \%$ de la capacité au champ. L'activité photosynthétique nette de Crau est alors divisée par 3, celle de Huia par 6, par rapport à leur témoin respectif.

\section{Fixation de l'azote}

L'évolution de l'ARA des témoins est stable et ne fait apparaître aucune différence significative entre les 4 cultivars. Au cours du déssèchement du sol, on observe 2 phases dans l'évolution de I'ARA (Fig. 3) :

- lors des premiers jours, on note une augmentation significative de l'ARA. Cet accroissement est lié à la diminution de la teneur en eau du sol, entraînant une meilleure aération du sol et par conséquent une stimulation de l'activité nitrogénase. Les niveaux de fixation d'azote les plus élevés sont ainsi obtenus pour des teneurs en eau du sol comprises entre 60 et $70 \%$ de la capacité au champ, soit une humidité pondérale de 16 à $18 \%$, pour les cultivars du type Ladino (Crau et Major), 3 jours après la supression de l'arrosage. Pour les variétés du type Hollandicum, l'optimum de fixation est déjà atteint le $2^{\mathrm{e}}$ jour; il se situe entre 80 et $85 \%$ de la capacité au 
Tableau I. Analyse de variance des mesures d'activité photosynthétique nette des 4 cultivars, Crau (CR), Major (MA), Luclair (LU) et Huia (HU), subissant (DEF) ou non (TEM) un déficit hydrique. Les moyennes suivies de la même lettre ne sont pas significativement différentes $(P=0,05)$ selon le test de Newman-Keuls.

\begin{tabular}{|c|c|c|c|c|c|c|c|}
\hline Temps (jour) & 1 & 2 & 3 & 4 & 5 & 6 & 7 \\
\hline \multicolumn{8}{|l|}{ Effet du facteur } \\
\hline $\begin{array}{l}\text { "déficit hydrique" } \\
\text { Témoin (TEM) }\end{array}$ & $\begin{array}{l}\text { NS } \\
61,34 a\end{array}$ & $\begin{array}{l}S^{\star \star \star} \\
72,30 a\end{array}$ & $\begin{array}{l}S^{\star \star \star} \\
65,47 a\end{array}$ & $\begin{array}{l}S^{75 x} \\
63,90 a\end{array}$ & $\begin{array}{l}S^{* 7 x x} \\
70,91 a\end{array}$ & & $60,65 a$ \\
\hline Déficit (DEF) & $59,92 a$ & $41,75 b$ & $31,78 \mathrm{~b}$ & $14,78 b$ & $9,80 \mathrm{~b}$ & & $1,83 b$ \\
\hline \multicolumn{8}{|l|}{ Effet du facteur intéraction } \\
\hline "déficit hydrique-cultivars" & $\mathrm{S}^{*}$ & NS & NS & $S^{* *}$ & NS & & NS \\
\hline TEM - CR & $61,54 a b$ & $73,61 a$ & 63,86 & $62,07 a$ & $72,58 \mathrm{a}$ & & $60,24 a$ \\
\hline TEM - MA & $65,75 \mathrm{a}$ & $67,64 a$ & 68,83 & $64,02 a$ & $71,48 a$ & & $60,96 a$ \\
\hline TEM - LU & $48,54 b$ & $77,54 a$ & 67,84 & $62,95 a$ & $74,44 a$ & & $62,46 a$ \\
\hline TEM - HU & $69,54 a$ & $70,42 a$ & 61,36 & $66,55 a$ & $65,14 a$ & & $58,94 a$ \\
\hline DEF - CR & $60,37 a b$ & $46,14 b$ & 32,93 & $22,26 b$ & $14,71 b$ & & $3,84 \mathrm{~b}$ \\
\hline DEF - MA & $58,38 a b$ & $42,11 \mathrm{~b}$ & 36,51 & $15,88 \mathrm{c}$ & $10,39 b$ & & $2,17 \mathrm{~b}$ \\
\hline DEF - LU & $62,34 a b$ & $39,50 \mathrm{~b}$ & 29,47 & $11,23 \mathrm{~cd}$ & $7,83 b$ & & $1,03 b$ \\
\hline DEF - HU & $58,60 a b$ & $39,23 b$ & 28,21 & $9,73 d$ & $6,29 b$ & & $0,29 b$ \\
\hline
\end{tabular}

NS $=$ Non significatif au risque $5 \% ; \mathrm{S}=$ Significatif au risque $5 \%\left({ }^{*}\right), 1 \%(* *), 0,1 \%(* * *)$.

champ, ce qui correspond à une humidité pondérale de 21 à $22 \%$. Après le 4 e jour, le déssèchement détermine un effet négatif très marqué sur l'ARA pour Luclair et Huia. Major a le taux de fixation qui se maintient le plus tardivement (jusqu'au $5^{\mathrm{e}}$ jour) au-dessus des témoins.

Crau présente une activité nitrogénase importante malgré le déficit. Après 7 jours, l'activité de Crau est encore égale à $50 \%$ de son témoin contre $10 \%$ pour Huia. Le classement des cultivars est alors identique à ceux observés précédemment : Crau > Major >Luclair > Huia.

\section{Matière sèche des parties aériennes}

Les mesures ont été réalisées le dernier jour de l'expérimentation (Tableau II). Crau est la variété qui produit le plus de biomasse de parties aériennes chez les plantes témoins (17g MS par pot contre $11 \mathrm{~g}$ pour Luclair). Le déficit hydrique a induit une réduction variable de l'accumulation de MS selon les cultivars. Cette diminution atteint $33 \%$ chez Major, alors qu'elle est plus modérée chez les autres variétés : $17 \%$ chez Crau et seulement $12 \%$ chez Huia et Luclair.

\section{Discussion et conclusion}

Nos résultats montrent clairement que l'alimentation hydrique conditionne le potentiel de fixation d'azote du trèfle blanc, qui ne s'exprime pas pleinement pour un sol à forte humidité (aération insuffisante) ou en cours de dessèchement.
Cette étude confirme aussi la liaison étroite entre le potentiel hydrique de base et le déficit en eau du sol (Fig. 4) déjà observée chez le trèfle blanc par Sanchez-Diaz et Sanchez-Marin (1974), Aparicio Tejo et al. (1980) et Guckert et Laperrière (1987).

Le déficit affecte rapidement l'assimilation photosynthétique sans pour autant entraîner de diminution concomitante de la fixation symbiotique de l'azote lors des 3 premiers jours d'expérimentation.

L'hypothèse de la remobilisation des réserves giucidiques au niveau du stolon peut raisonnablement être avancée pour rendre compte d'une allocation de substrats carbonés aux nodosités, suppléant la chute de l'activité photosynthétique. La fixation est maximale pour un taux d'humidité du sol correspondant à $2 / 3$ de la capacité au champ pour le type Ladino et à $4 / 5$ pour le type Hollandicum. Des observations similaires ont été signalées pour le trèfle blanc par Laperrière (1984) sur un sol sableux, dans le cas du trèfle violet par Holter (1978) et chez la luzerne par Wahab et Zahran (1983).

En début d'expérience et dans ce type de sol, à la capacité au champ, les nodosités ne seraient pas à même d'exprimer leur potentiel de fixation par une insuffisance d'aération du sol. L'oxygène serait le facteur limitant de la fixation pour des humidités du sol voisines de la capacité au champ.

Albrecht et al. (1984) ont montré un effet du dessèchement sur le statut hydrique des nodosi- 
A.R.A. $\left(10^{-6}\right.$ mole $\left.\cdot h^{-1} \cdot g^{-1} M S\right)$

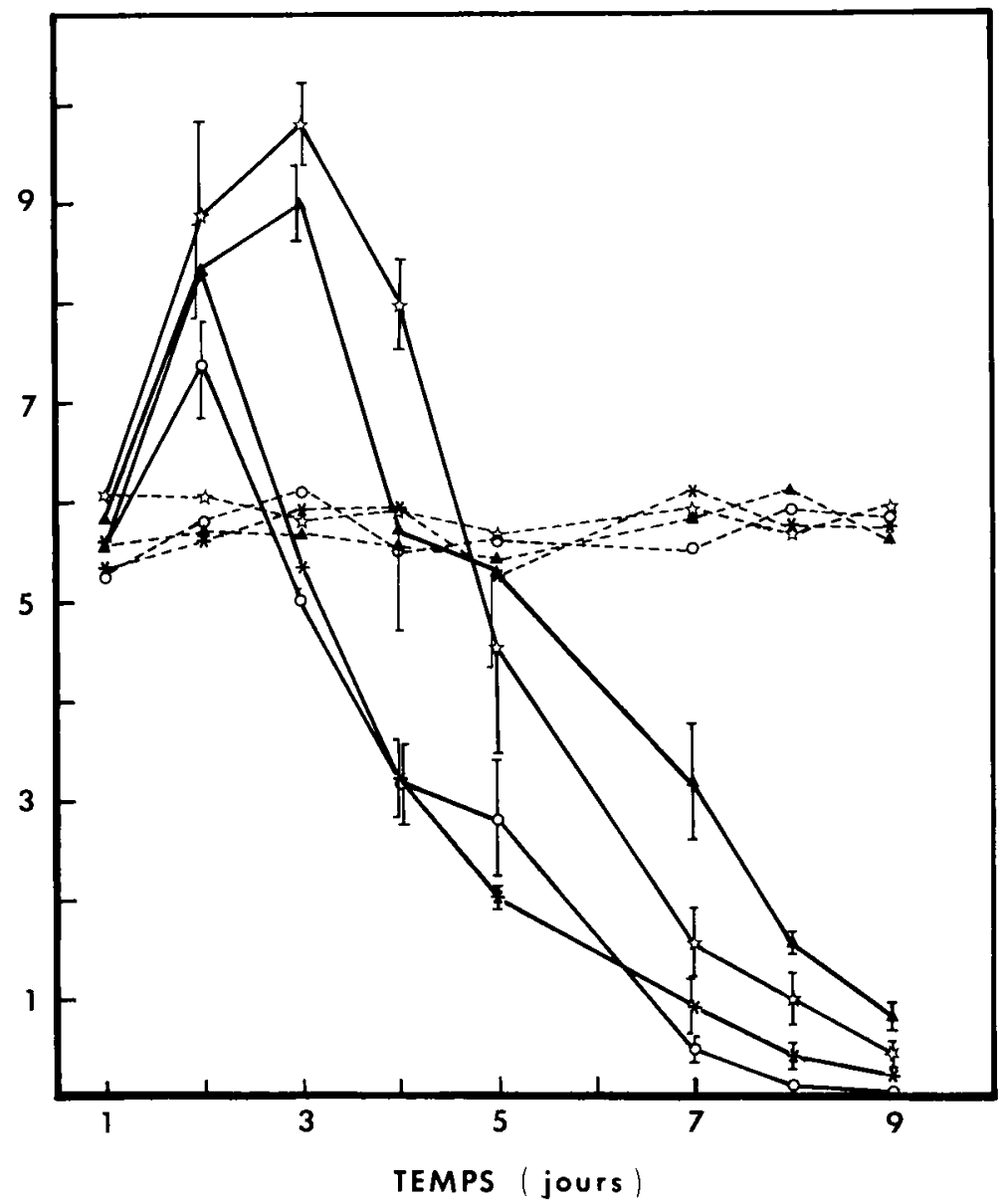

Fig. 3. Evolution de l'activité réductrice d'acétylène $\left(10^{-6} \mathrm{~mol} \mathrm{C}_{2} \mathrm{H}_{2} \cdot \mathrm{h}^{-1} \cdot \mathrm{g}^{-1} \mathrm{M} . \mathrm{S}\right.$. de parties aériennes) en fonction du temps (jours) pour 4 cultivars de tréfle blanc, (Crau : $\boldsymbol{\Lambda}$, Major : $\downarrow$, Luclair : * et Huia : 0 ) subissant $(-)$ ou non $(--\rightarrow$ un déficit hydrique. La première mesure a été effectuée $8 \mathrm{~h}$. après le dernier arrosage des pots soumis au déficit. Les traits verticaux correspondent à l'écart type des mesures.

Tableau II. Masses de matières sèches des parties aériennes des 4 cultivars en fin de déficit : feuilles, stolons, feuilles + stolons ( $\mathrm{g} \mathrm{MS}$. pot-1) et masse en \% du témoin.

\begin{tabular}{|c|c|c|}
\hline Feuilles (F) & Stolons (S) & $\begin{array}{l}\text { Total } \\
(F+S)\end{array}$ \\
\hline
\end{tabular}

\begin{tabular}{llrrrr}
\hline \multirow{2}{*}{ Crau } & Tém. & 12,6 & 4,3 & 16,9 & \\
& Déf. & 10,2 & 3,8 & 14,0 & 82,8 \\
Huia & Tém. & 11,8 & 2,7 & 14,5 & 87,6 \\
& Déf. & 9,6 & 3,1 & 12,7 & \\
\multirow{4}{*}{ Luciair } & Tém. & 8,5 & 2,4 & 10,9 & 88,1 \\
& Déf. & 7,5 & 2,1 & 9,6 & \\
\multirow{2}{*}{ Major } & Tém. & 10,6 & 3,2 & 13,8 & 67,4 \\
& Déf. & 7,4 & 1,9 & 9,3 & \\
\hline
\end{tabular}

tés, avec une déshydratation et une altération des tissus, sans que ces phénomènes soient en relation directe avec des variations des niveaux de l'activité photosynthétique. Selon Sprent
(1972), le déficit hydrique a un rôle direct sur l'activité des nodosités, et cet effet serait amplifié par la réduction du transport d'assimilats aux nodosités à partir des feuilles. 
CAPACITE AU CHAMP $\%$

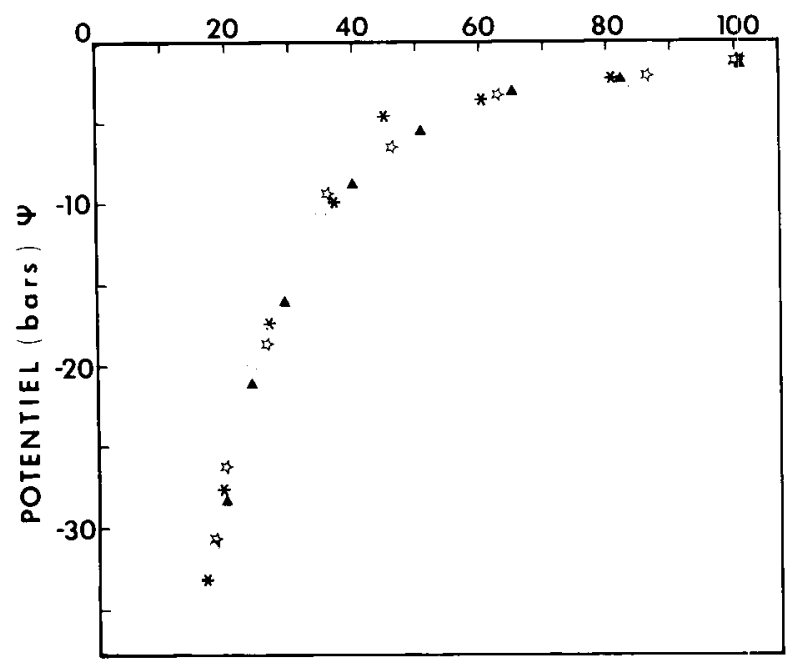

Fig. 4. Relation entre la teneur en eau du sol, exprimée erı \% de la capacité au champ, et le potentiel hydrique de base dans le cas des plantes soumises au déficit hydrique. Crau (A), Major (约), Luclair (解), Huia (O).

Dans le cas d'un déficit modéré, Sprent (1976) note qu'une augmentation de la pression partielle en $\mathrm{O}_{2}$ rétablit la fixation; les premiers effets du déficit seraient de diminuer les prélèvements d'oxygène par les nodosités, réduisant d'autant le niveau des métabolites (ATP) utilisés pour la fixaton. Pankhurst et Sprent (1975) discutent d'un possible développement d'une barrière à la diffusion des gaz et/ou d'une diminution de l'affinité de la léghémoglobine pour l'oxygène lors d'un déficit hydrique.

Par ailleurs, Minchin et al. (1983) ont montré un rôle inhibiteur de l'acétylène (utilisé dans les mesures d'ARA) sur l'activité fixatrice des nodosités; une augmentation de la résistance à la diffusion des gaz au niveau des nodosités serait induite par l'acétylène et créerait une diminution des flux d' $\mathrm{O}_{2}$ vers les bactéroïdes (Sheehy et al., 1983; Witty et al., 1984). Il est important de noter qu'après une semaine de déficit hydrique les 4 cultivars testés maintiennent encore une activité physiologique, certes faible, mais toujours mesurable. Les différences variétales sont importantes et significatives quel que soit le paramètre en jeu (potentiel hydrique de base, assimilation photosynthétique nette, ARA). Ces expériences mettent en évidence l'intérêt de déterminer le potentiel hydrique de base qui permet de différencier non seulement les types, mais aussi les cultivars. Ainsi, à cet égard, le type Hollandicum (et notamment Huia) est plus sensible à une alimentation hydrique déficitaire que le type Ladino (Major et surtout Crau).
La gestion de l'eau moins économique du type Hollandicum n'est pas due à une masse foliaire plus grande (Tabl. II), qui serait à l'origine d'une consommation d'eau plus élevée, mais elle serait plus vraisemblablement en relation avec la perméabilité foliaire (peut-être dimension et régulation stomatiques). Le mode de fonctionnement hydrique se répercute sur les taux de fixation d'azote et d'assimilation du carbone. Mais il est à remarquer que le classement des génotypes suivant leur réponse à la sécheresse, en prenant ces critères comme référence, diffère sensiblement de leur classement quand on considère la réduction relative de production de matière sèche, en condition d'alimentation hydrique déficitaire (Tabl. II). Par exemple, la variété Major subit une plus forte réduction de croissance que les 3 autres variétés, alors que son fonctionnement sur les plans hydrique, azoté et carboné apparaît tout à fait satisfaisant.

Ces données montrent clairement la décroissance de la fixation lors d'un déficit hydrique; elles nécessitent cependant d'être approfondies par une approche plus fine des mécanismes en jeu. Des études en cours de réalisation cherchent à établir, notamment au niveau des nodosités, les relations entre activité nitrogénase et processus physiologiques induits par le déficit en eau.

\section{Références}

Albrecht S.L., Benneth J.M. \& Boote K.J. (1984) Relationship of nitrogenase activity to plant water stress in field grown soybeans. Field Crops Res. 8, 1-2, 61-71

Aparicio-Tejo P.M., Sanchez-Diaz M.F \& Pena J.I. (1980) Nitrogen fixation, stomatal response and transpiration in Medicago sativa, Trifolium repens and Trifolium subterraneum under water stress and recovery. Physiol. Plant. 48,1-4

Aussenac G. \& Granier A. (1978) Quelques résultats de cinétique journalière du potentiel de sève chez les arbres forestiers. Ann. Sci. For. 35, 1, 19-32

Balandreau J. \& Dommergues Y. (1973) Assaying nitrogenase $\left(\mathrm{C}_{2} \mathrm{H}_{2}\right)$ activity in the fields. Bull. Ecol. Res. Comm. NFR 17, 247-254

Damay J. (1981) Contribution à l'étude de la fixation d'azote par le trèfle blanc (Trifolium repens L.) : aspects écologiques et agronomiques. Thèse Docteur Ingénieur INPL

Engin M. \& Sprent J.I. (1973) Effects of water stress on growth and nitrogen fixing activity of Trifolium repens. New Phytol. 72, 117-126

Foulds W. (1978) Response to soil moisture supply in three leguminous species. New Phytol. 80, 547-555

Guckert A., Damay J., Treillet L., Balandreau J., Bardin R. \& Chalamet A. (1983) Etude au champ de la fixation d'azote par le trèfle blanc (Trifolium repens L.). Fourrages 94, 61-86

Guckert A. \& Laperrière C. (1987) Effet du stress 
hydrique sur la fixation d'azote par le trèfle blanc (Tr $i$ folium repens L.). Coll. INRA, 37, 245-250

Holter V. (1978) Nitrogen fixation of 4 legumes in relation to aboveground biomass, root biomass, nodule number and water content of the soil. Oikos 31, 230235

Koch B. \& Evans H.J. (1966) Reduction of acetylene to ethylene by soybean root nodules. Plant Physiol. 47, 453-456

Laissus R. (1981) Ajustement de la fertilisation azotée des prairies pâturées en vue d'utiliser les potentialités du trèfle blanc. C.R. Seances Acad. Agric. Fr. 67, 599615

Laperrière C. (1984) Etude de la fixation d'azote par le Trèfle blanc. Aspects biologiques et agronomiques. Thèse INPL, ENSAIA, Nancy

Minchin F.R., Witty J.F., Sheehy J.E. \& Muller M. (1983) A major error in the acetylene reduction assay : decreases in nodular nitrogenase activity under assay conditions. J. Exp. Bot. 34, 641-649

Pankhurst C. E. \& Sprent J.I. (1975) Effects of water stress on the respiratory and nitrogen-fixing activity of soybean root nodules. J. Exp. Bot. 26, 287-304

Ritchie G.A. \& Hinckley T.M. (1975) The pressure chamber as an instrument for ecological research. Adv. Ecol. Res. 9,165-254
Sanchez-Diaz M. \& Sanchez-Marin M. (1974) Resistencia a la desecación del tejido foliar y cierre de estomas en alfalfa (Medicago sativa $L$.) y trebol blanco (Trifolium repens L.) con relación al deficit de agua. An. Edafol. Agrobiol. 33, 743-754

Scholander P.F., Hammel H.T., Bradstreet E.D. \& Hemmingsen E.A (1965) Sap pressure in vascular plants. Science 148, 339-346

Sheehy J.E., Minchin F.R. \& Witty J.F. (1983) Biological control of the resistance to oxygen flux in nodules. Ann. Bot. 52, 565-571

Sprent J.I. (1972) The effects of water stress on nitrogen fixing root nodules. Il. Effects on the fine structure of detached soybean nodules. New Phytol. 70, 9-17

Sprent J.I. (1976) Water deficit and nitrogen-fixing root nodules. In : Water Deficit an Plant Growth, Academic Press, New York, pp. 291-315

Wahab A.M.A. \& Zahran H.H. (1983) The effect of water stress on $\mathrm{N}_{2}\left(\mathrm{C}_{2} \mathrm{H}_{2}\right)$ fixation and growth of Medicago sativa L.. Acta Agron. Acad. Sci. Hung. 32, 1-2, 114-118

Witty J.F, Minchin F.R., Sheehy J.E \& Minguez M.I. (1984) Acetylene-induced changes in the oxygen diffusion resistance and nitrogenase activity of legume root nodules. Ann. Bot. 53, 13-20 